\title{
Las redes informales inter-oganizacionales en la movilización por el agua en la Cuenca de México, 2005-2009
}

\author{
Leonardo Garavito $\mathrm{G}^{1}$.- El Colegio de México A.C. - Universidad Externado de \\ Colombia
}

\begin{abstract}
Resumen
Este artículo presenta un análisis de las redes informales inter-organizacionales tanto al interior de la movilización por el agua en la Cuenca de México como con su contexto organizativo. Estas redes se basan en vínculos de cooperación y de reconocimiento básicos para el desarrollo y el mantenimiento de los procesos colectivos de un movimiento social. Dichos procesos incluyen la producción de identidades comunes, de significados compartidos y la realización de acciones colectivas. Los resultados principales indican que la movilización cumple con las características estructurales básicas para configurar un movimiento social con vocación de permanencia frente al orden social actual en materia del agua en la región.
\end{abstract}

Palabras clave: Redes inter-organizacionales, Movimientos sociales, Agua, Cuenca de México.

\begin{abstract}
This paper presents a social network analysis within the water social movement organizations at México's basin, and also with movement's organitazional context. These networks are inter-oganizational cooperation and appreciation ties based. These relations represent some of the foundamental collective procceses for social movement development and survival. These processes include share identities and meanings production and collective's action movilization. Finally, this paper concludes that water social movement at Mexico's basin fulfill the basics structural contiditions as a long term vocation social movement.
\end{abstract}

Key words: Inter-organizaciotal networks, Social movements, Water, Mexico's basin.

\footnotetext{
1 Ph. D. en Estudios Urbanos y Ambientales. Investigador y Docente de la Universidad Externado de Colombia. Este texto se deriva la tesis doctoral: "Un Oasis en el desierto. Reflexividad y redes sociales por el agua en la Cuenca de México, 2005-2009". Correos electrónicos de contacto: leonardogaravito@hotmail.com, Igaravito@colmex.mx, luis.garavito@uexternado.edu.co
} 


\section{Introducción}

El objetivo de éste artículo es analizar las redes informales tanto al interior de la movilización por el agua en la Cuenca de México como con su contexto organizativo. De acuerdo a Diani (2003) las redes informales son unos mecanismos de coordinación entre los actores sociales, donde los términos de intercambio, la distribución de las tareas y el reconocimiento dependen exclusivamente de las partes involucradas. Estas redes se basan en vínculos de cooperación y de reconocimiento necesarios para articular los procesos colectivos básicos en el desarrollo o el mantenimiento de un movimiento social. Estos procesos incluyen la generación de identidades comunes, de significados compartidos y la realización de acciones colectivas. Dado lo anterior, el análisis de las redes informales es un enfoque teórico reciente en el estudio de las movilizaciones sociales, que consiste en identificar e interpretar los patrones relacionales de una movilización.

Para tal efecto, el contenido de este texto se divide en tres secciones: en la primera se presentan los elementos teóricos y metodológicos utilizados para el análisis de las redes informales. En la segunda, se desarrolla el análisis de estas redes al interior de la movilización. Y en la tercera, se presenta el análisis de las redes entre las organizaciones de la movilización y su contexto organizativo.

A manera de resumen, los principales resultados de este capítulo indican que:

En primer lugar: al interior de la movilización se evidencia una red informal que tiende a acerar a las organizaciones entre sí y que facilita su desarrollo como un movimiento social. Su estructura revela una complementariedad entre las organizaciones que ocupan las posiciones estructurales centrales y las periféricas. En efecto, mientras las primeras son las fuentes principales de integración y sirven como intermediarias; las segundas, aportan los suficientes vínculos periféricos para evitar que la integración dependa de unos pocos actores centrales. Esta combinación, se traduce en una estructura relacional que tiende a ser incluyente y horizontal, en un contexto de múltiples liderazgos.

En este sentido, la principal influencia de las organizaciones más centrales sobre el desarrollo de movilización consiste en proponer 'el derecho humano al agua' como el marco de interpretación frente a las diversas problemáticas del agua en la región. Otras influencias centrales son favorecer el intercambio abierto de información, apoyar la generación y la difusión de tecnologías ecológicas para el uso del agua, y estimular el desarrollo de modelos comunitarios para el manejo del agua y de los recursos naturales. En conjunto, las organizaciones más centrales se enfocan tanto 
en la defensa del derecho humano al agua y la búsqueda de incidencia sobre las políticas públicas; como en promover un impacto cultural directo sobre la población, por medio de procesos de apoyo y de capacitación sobre diversas prácticas sustentables en relación con el uso del agua.

De otra parte, las organizaciones periféricas aportan un carácter pluralista e incluyente a la movilización al introducir algunos temas y enfoques alternativos. En entre estos se incluyen: el fortalecimiento de los procesos de participación social, el desarrollo urbano sostenible, la lucha por la prestación y el acceso adecuado los servicios públicos, la equidad de género, e incluso, una marco interpretativo distinto al 'derecho humano al agua' basado en una perspectiva ecocéntrica, según el cual, ni los seres humanos y ni sus necesidades poseen una posición privilegiada en comparación con el resto de seres vivos.

En segundo lugar: las redes entre la movilización y su contexto organizativo indican que la movilización cuenta con un contexto compartido, con el cual se integra por medio de múltiples vínculos. El contexto organizativo se conforma por 143 actores que incluyen organizaciones sociales, privadas e instituciones públicas, tanto nacionales como internacionales. De manera principal, la movilización tiende a acercarse hacia otras organizaciones sociales nacionales e internacionales y hacia un conjunto de instituciones públicas nacionales.

Dicha dualidad evidencia dos procesos paralelos en la movilización: primero, un proceso de identificación y de fortalecimiento de las relaciones con otras organizaciones sociales nacionales e internacionales. Dicho proceso estimula a las organizaciones a definirse y percibirse como participantes de un movimiento social por el agua de alcance no solo local, sino nacional y global. Y segundo, un proceso de acercamiento frente a las autoridades públicas nacionales, estatales y locales, basado en el desarrollo de vínculos de cooperación y de reconocimiento. Esto implica que la movilización además de promover un orden social alternativo en función del cual enfrenta y crítica el modelo dominante, también tiende a cooperar con las autoridades gubernamentales de manera estratégica de acuerdo a sus objetivos propios. Sin embargo, dicha cercanía entre la movilización y las autoridades deja plantea una cuestión relevante: ¿se está comprometiendo la capacidad crítica y el rol de control político que caracterizan a los movimientos sociales, en la medida en la movilización tiende a aliarse y cooperar de manera creciente con las autoridades gubernamentales? 
En menor medida pero también relevante, la movilización tiende a acercarse tanto al sector académico nacional como a algunas organizaciones privadas. Los vínculos con el sector académico inciden directamente sobre los procesos colectivos relacionados con la construcción de significados compartidos. Entre sus aportes esta la información y el análisis de la problemática local, nacional e internacional; además de la realización de eventos que reúnen a diversos actores sociales y fomentan el debate y la difusión de la información sobre el tema del agua.

Por último, los vínculos con las organizaciones privadas tienden a configurar una influencia controversial al interior de la movilización en tanto que, de una parte, representan a grupos comerciales que apoyan el manejo mercantil del agua pero que; de otra parte, ofrecen recursos económicos y financieros para la ejecución de múltiples proyectos sociales y ambientales, a través de sus programas y sus obligaciones de responsabilidad social y ambiental.

En suma, es posible afirmar que el desarrollo de la movilización contemporánea por el agua en la Cuenca de México se sustenta en una serie de redes informales, tanto en su interior como en su exterior, capaces de sostener y estimular los procesos colectivos que fundamentan un movimiento social. Por lo tanto, la movilización cumple con las características estructurales básicas para configurar un movimiento social con vocación de permanencia, el cual busca instaurar diversas transformaciones tanto políticas como culturales frente al orden social actual en materia del agua. No obstante, los principales retos que afectan la consistencia de la movilización se relacionan con una cercanía demasiado intensa con el sector gubernamental, que derive en la cooptación de una parte de la movilización; o en la pérdida de identidad común y el enfrentamiento interno en relación a la relevancia de las organizaciones privadas que representan a la interpretación mercantil del agua.

\section{Elementos teóricos y metodológicos para el análisis de las redes informales}

¿La movilización por el agua en la Cuenca de México puede considerarse como un 'movimiento social'? ¿Qué puede ser considerado como un movimiento social? y ¿Qué diferencia un movimiento social de otros fenómenos sociales? son la preguntas claves que ordenan esta reflexión.

Para comenzar, un movimiento social se puede definir como un grupo de actores que persiguen algún tipo de transformación del orden social establecido, en el marco de un Estado Nacional. Para esto, cuentan con un repertorio de acciones 
conflictivas, como marchas, protestas, manifestaciones, boicots, levantamientos, acciones legales, entre otras, con las cuales intentan conseguir sus objetivos (Tilly, 1978). Un ejemplo son las diversas luchas de los siglos XIX y XX por el reconocimiento de los derechos laborales y por los derechos civiles. De acuerdo a esta definición, la relación entre los movimientos sociales y el Estado es caracterizada por un conflicto frente al orden social. Desde esta óptica, los movimientos sociales son rivales del Estado ya que son capaces de mostrar las debilidades de las élites y las autoridades (Tarrow, 2004).

No obstante, dicha rivalidad es considerada como estratégica y no excluye a la colaboración. En este sentido, los movimientos sociales se caracterizan no solo por una oposición férrea al Estado, sino también por reaccionar en función de la estructura de oportunidades que le brinda el sistema político (Tarrow, 2004). En algunos casos, los movimientos sociales y el Estado trabajan como aliados en temas de interés común como el medio ambiente, las inequidades o las tragedias sociales.

De forma adicional, fenómenos como la globalización y la ampliación de la democracia han estimulado la formación de movimientos sociales transnacionales y con vocación de permanencia. De acuerdo a Melucci (1999: 97) "el sistema social adquiere una dimensión planetaria y los acontecimientos no son importantes en sí mismos (...) sino por su impacto simbólico en el sistema mundial". Así, además del impacto político local de los movimientos sociales, éstos se caracterizan por tener una dimensión global y simbólica al ofrecer formas de pensar y de actuar alternativas al orden social.

En suma, un movimiento social se define como un grupo de actores sociales que persiguen algún tipo de transformación del orden establecido, en el marco de un sistema planetario. Para lo cual cuentan con un repertorio de acciones conflictivas, de redes sociales y un valor simbólico, que los vinculan de forma estratégica tanto a la sociedad global como al Estado nacional en los contextos locales. No obstante, esta definición no es exhaustiva sobre las particularidades estructurales que diferencian a los movimientos de otros fenómenos sociales colectivos. En efecto, para pasar de una definición más teórica a una más procedimental, es necesario identificar cuáles son las particularidades organizativas que caracterizan a los movimientos sociales.

Según Diani (2003) el análisis de redes sociales sirve para caracterizar las estructuras que subyacen a los intercambios materiales o simbólicos que interconectan a un grupo de actores sociales. Desde el punto de vista relacional, propone que los movimientos sociales 
consisten en actores formalmente independientes, imbuidos en un contexto local (donde 'local' delimita un espacio social o territorial), que tienen identidades, valores, orientaciones y objetivos específicos, pero que al mismo tiempo se vinculan a través de diversas formas de cooperación concreta y/o de reconocimiento mutuo en una relación que se extiende más allá cualquier protesta o campaña específica (Diani: 301).

De tal forma, los vínculos de cooperación concreta y de reconocimiento mutuo entre los actores sociales son los elementos básicos que estructuran el desarrollo de un movimiento social. A su vez, los múltiples vínculos configuran las redes sociales informales que funcionan como mecanismos de coordinación, sosteniendo los procesos colectivos como el desarrollo de identidades y significados compartidos, y la realización de las acciones colectivas.

Así, para saber en qué medida la movilización por el agua en la Cuenca de México puede definirse como un movimiento social, se analizan los vínculos de colaboración y de reconocimiento en su interior y en relación con su entorno organizacional. La información utilizada fue recabada a través de una serie de entrevistas aplicadas a un grupo de informantes clave. Este grupo fue conformado por representantes de 18 organizaciones sociales con diversos intereses sobre el agua en la CM. La selección de las organizaciones partió de una identificación de 24 participantes potenciales, que se redujo en función de su disponibilidad. Es decir, solo aquellas que aceptaron participar voluntariamente como informantes clave del proyecto. El listado de las organizaciones entrevistadas presenta en la tabla a continuación, cada una se posee un número que sirve para identificar su lugar en las representaciones gráficas de las redes más adelante. El periodo de estudio que abarca desde 2005 al 2009 permite capturar una imagen de las relaciones recientes más relevantes y representarlas como un conjunto de redes sociales.

\begin{tabular}{|c|c|}
\hline No. & Nombre \\
\hline 1 & Fondo para la Comunicación y la Educación Ambiental A.C. \\
\hline 2 & Instituto Internacional de Recursos Renovables A.C. -México. \\
\hline 3 & Red Ecologista Autónoma de la Cuenca de México - Ecocomunidades. \\
\hline 4 & Consejo Consultivo del Agua A.C. \\
\hline 5 & $\begin{array}{l}\text { COMDA. Coalición de Organizaciones Mexicanas por el Derecho al } \\
\text { Agua. }\end{array}$ \\
\hline 6 & PRONATURA A.C. \\
\hline 7 & Presencia Ciudadana Mexicana A.C. \\
\hline 8 & CEMDA A.C. Centro Mexicano de Derecho Ambiental. \\
\hline 9 & Patronato Pro Zona Mazahua A.C. \\
\hline 10 & Red Mexicana de Acción por el Agua. \\
\hline 11 & CEJA A.C. Centro de Estudios Jurídicos Ambientales. \\
\hline 12 & Fundación Tlaloc A.C. \\
\hline 13 & Guardianes de los Volcanes A.C. \\
\hline
\end{tabular}




\begin{tabular}{|l|l|}
\hline 14 & UPREZ. Unión Popular Revolucionaria Emiliano Zapata. \\
\hline 15 & Asistencia Legal por los Derechos Humanos - ASILEGAL A.C. \\
\hline 16 & Mujer y Medio Ambiente A.C. \\
\hline 17 & Equipo Pueblo A.C. \\
\hline 18 & Fondo Pro-Cuenca del Valle de Bravo A.C. \\
\hline
\end{tabular}

Tabla 1. Organizaciones de la movilización.

En cuanto a los vínculos, en primer lugar, los de colaboración reflejan el esfuerzo invertido por las organizaciones para realizar acciones colectivas y compartir información. En efecto, evidencian diversas manifestaciones de cooperación concreta entre las organizaciones de la movilización, y entre éstas y su contexto organizativo. En segundo lugar, los vínculos de reconocimiento representan la cercanía entre las organizaciones en términos perceptuales. Es decir, corresponden a una identificación subjetiva sobre quiénes son sus las principales fuente de apoyo o los aliados. En conjunto, dichos vínculos representan una estructura de la cercanía entre los diversos actores sociales tanto interior como en el contexto externo de la movilización. De hecho, la relevancia de la cercanía se asocia con

un sentido de propósitos comunes y de compromisos frente a una causa, que permite a los activistas solitarios y a las organizaciones observarse como intrincadamente vinculados, no necesariamente idénticos pero compatibles, en una movilización más amplia (Diani, 2004:284)

Para el análisis de las relaciones al interior de la movilización se generaron tres redes: primero, la red de interacciones 2005-2009 basada en los vínculos producto de la realización de acciones colectivas o el intercambio de información. Segundo, la red de valoraciones 2005-2009 basada en los vínculos subjetivos de reconocimiento de apoyos y aliados. Y por último, la red general de cercanía 2005-2009 que combina las dos anteriores. El análisis estructural de dichas redes abarca su densidad, sus componentes principales y la distribución estructural de sus actores.

Primero, la densidad indica la proporción de vínculos existentes en relación al total de los vínculos potenciales en la red. En este sentido, una densidad del $0 \%$ significaría que no existe ningún vínculo entre los actores, mientras que el 100\% implica que cada uno se encuentra conectado con todo el resto. En cuanto a los movimientos sociales, un alto grado densidad señala la existencia de vínculos redundantes y una muy fuerte afinidad ideológica entre los actores. Esta condición se relaciona con los momentos más álgidos de los ciclos de protesta. Por el contrario, una muy baja densidad indica la preeminencia de estrategias de alianzas cerradas entre grupos aislados de pocas organizaciones, o que solo realizan pequeñas colaboraciones en temas específicos (Diani, 2003). 
Segundo, el análisis de los componentes permite evaluar la conectividad general de la red al identificar y caracterizar la cantidad y tipo de subgrupos que la componen. Esta herramienta es útil para diferenciar fracturas al interior de la movilización que promuevan diversas tendencias ideológicas o de acción.

Y tercero, la distribución estructural de los actores permite identificar cuáles son los actores más y menos centrales o periféricos. Con esto se caracteriza y se diferencia su poder relacional, su rol y su impacto particular sobre las redes. En efecto, la posición estructural determina el control efectivo y potencial de los actores sobre los recursos relacionales, materiales o simbólicos, que fluyen por las redes; además, refleja su posicionamiento en relación con su prestigio y expansividad en las redes (Wasserman y Faust, 1994). En este sentido, permite caracterizar cómo se distribuye el poder relacional en relación con el desarrollo de los procesos colectivos de los movimientos sociales. Este análisis se basa en los indicadores de centralidad de grado nodal, los cuales representan en términos absolutos la cantidad de vínculos que posee cada organización, y en términos relativos el porcentaje de la movilización que se encuentra vinculada con cada organización. Vale la pena aclarar que no se discrimina entre vínculos de salida y de entrada, ya que no se realizan análisis más detallados sobre la centralidad por popularidad (vínculos de entrada) o por expansión (vínculos de salida); sin embargo, los vínculos en general, más allá de su direccionalidad, permiten identificar e incluir algunos actores o relaciones omitidas voluntaria o involuntariamente por algunos de los informantes, al ser declaradas por sus contrapartes.

De otra parte, para el análisis de los vínculos entre la movilización y su contexto organizativo se incluye una red adicional: La red general de cercanía con el contexto organizativo 2005-2009. El estudio de esta red permite caracterizar la permeabilidad de la movilización en relación a su contexto, e identificar cuáles son las principales influencias externas sobre sus procesos colectivos. Para esto, se identifican diversos sectores sociales más o menos cercanos a la movilización, divididos en seis grupos: 1 . organizaciones sociales nacionales, 2. Organizaciones sociales internacionales, 3. Instituciones gubernamentales nacionales, 4. Instituciones gubernamentales internacionales, 5. Organizaciones privadas y, 6 . Instituciones educativas. Al interior de estos subgrupos, se identifican cuáles son los actores más centrales y cómo es su impacto potencial sobre los procesos colectivos de la movilización. 
A manera de cierre, el análisis de los vínculos con el contexto organizativo permite revisar los límites de la movilización tanto en tamaño como de enfoque. En cuanto al tamaño, permite identificar actores del contexto tan centrales y afines a la movilización que deberían considerarse como parte integral de esta. Y en términos de enfoque, puesto que refleja hacia qué sectores sociales se enfocan de manera principal sus acciones y reconocimientos y, por lo tanto, cómo éstos pueden llegar a influenciar el devenir de la movilización.

\section{Las redes informales al interior de la movilización}

Para comenzar, se presentan los grafos que representan los vínculos de cooperación y de reconocimiento al interior de la movilización: en el gráfico 1 , la red de interacciones 2005-2009 basada en la realización de acciones colectivas y el intercambio de información. En el gráfico 2, la red de valoraciones 2005-2009 que refleja los reconocimientos de apoyos y de alianzas. $Y$ en el grafo 3 , la red general de cercanía 2005-2009 que agrega a las dos anteriores. En cada gráfica los puntos rojos simbolizan a las organizaciones de la movilización identificadas con números de acuerdo a la tabla 1 , y las líneas representan los vínculos planteados anteriormente.

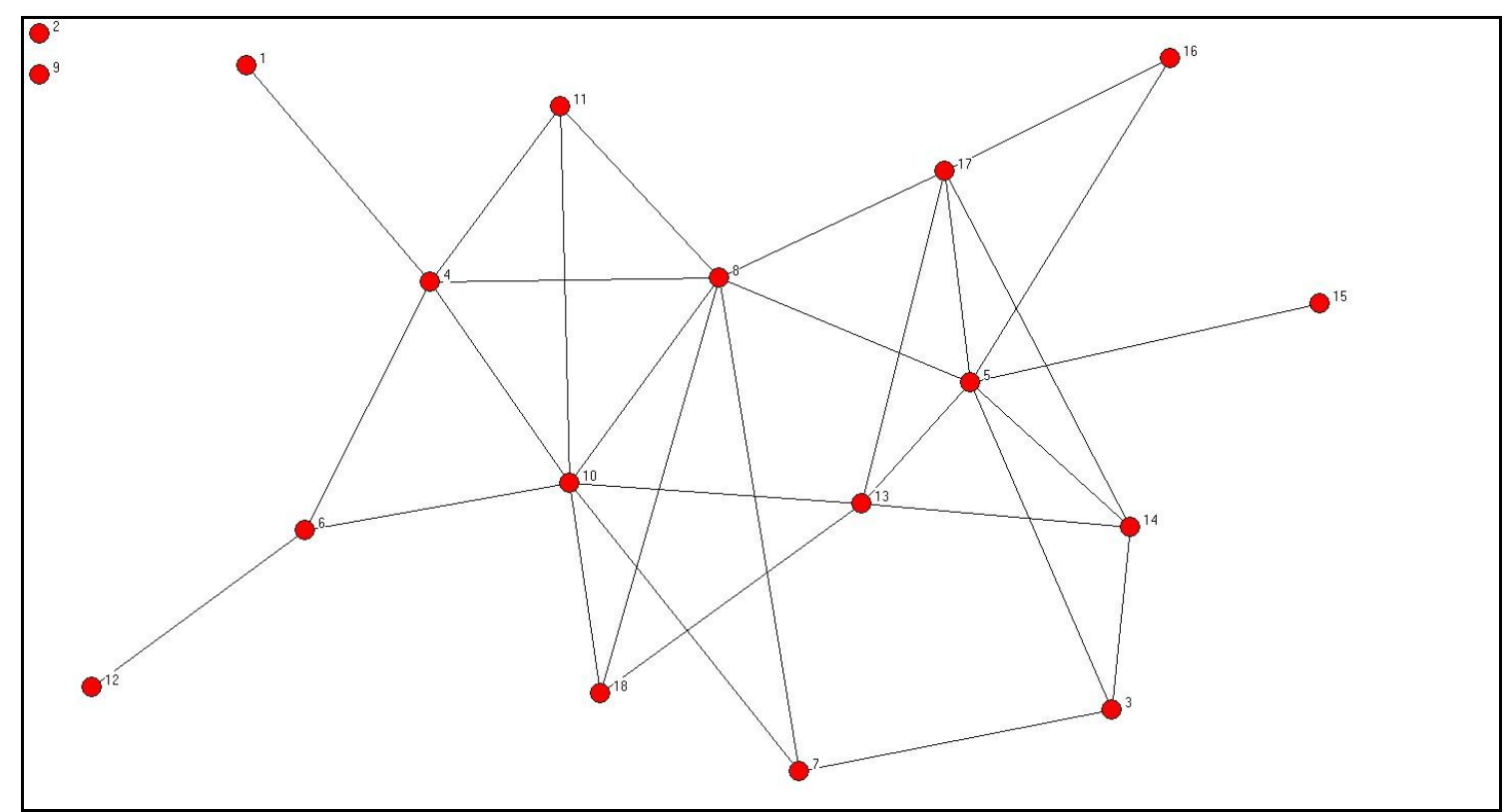

Gráfico 1. Red de interacciones 2005-2009. 


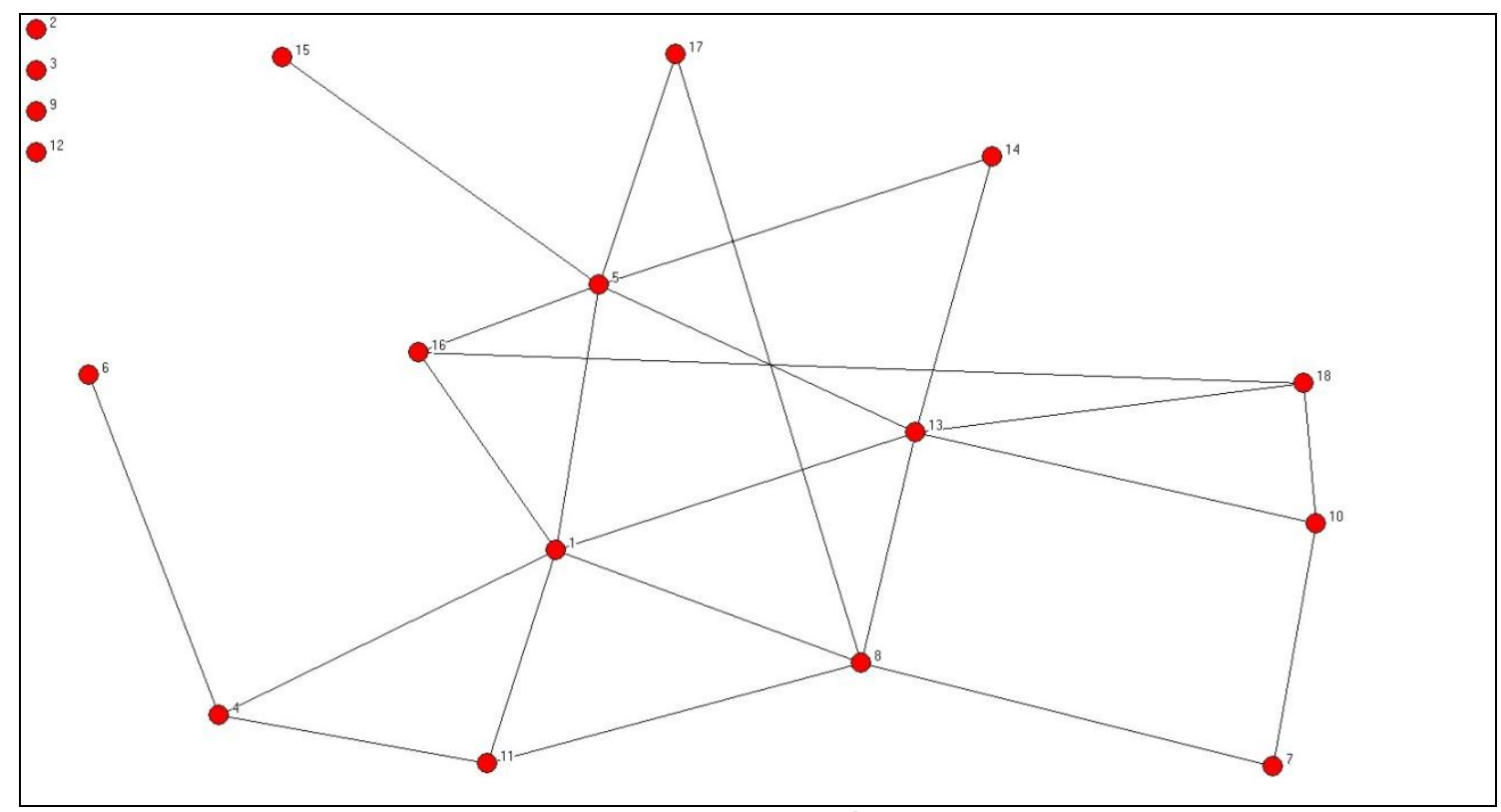

Gráfico 2. Red de valoración 2005-2009.

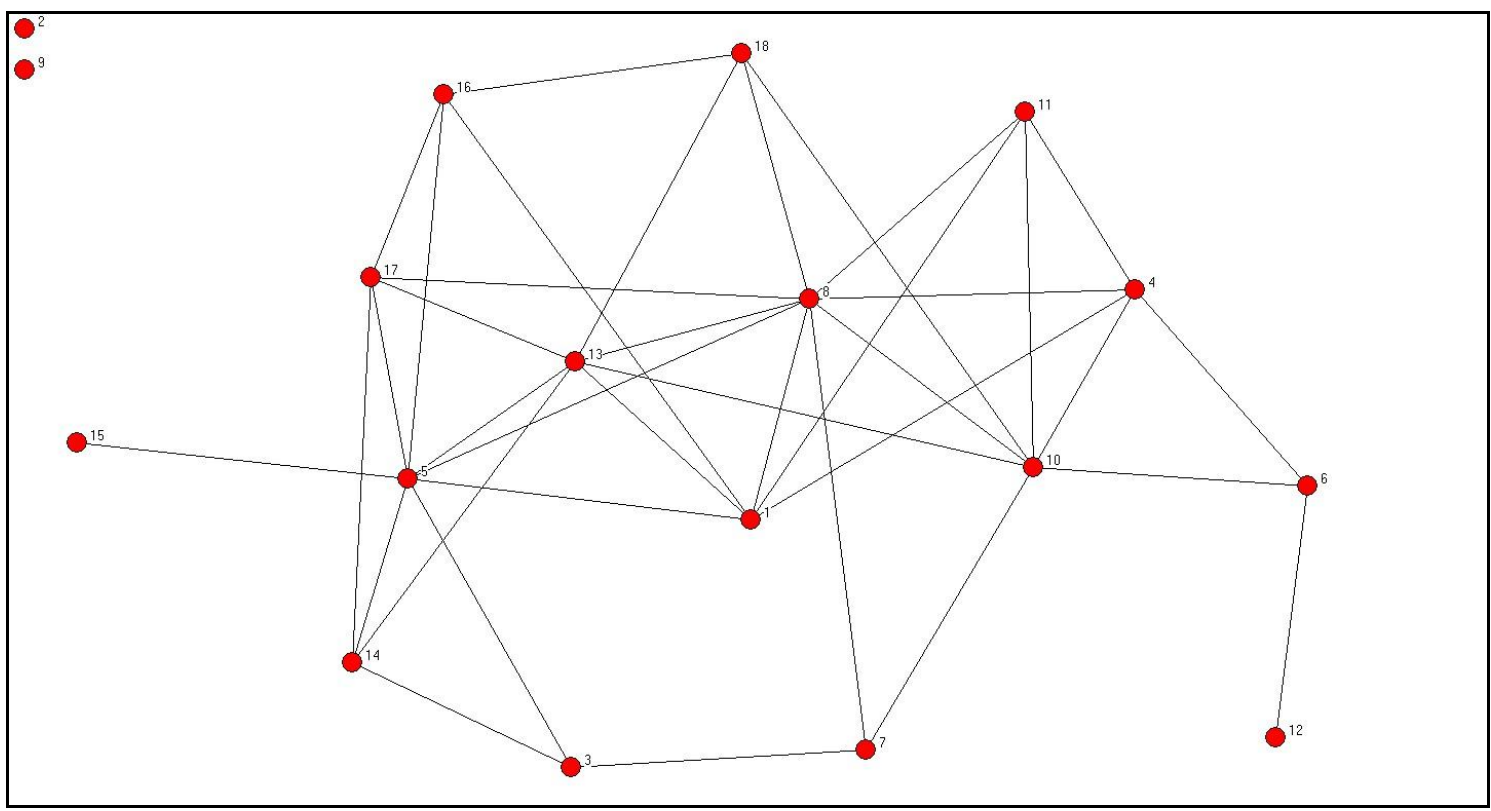

Gráfico 3. Red general de cercanía 2005-2009.

Los anteriores grafos evidencian la existencia de una red informal que incluye relaciones tanto de cooperación como de reconocimiento entre las organizaciones de la movilización. En efecto, cada una de las redes tiende a interconectar entre sí a la mayor parte de sus actores. En este sentido, respaldan la conclusión preliminar de que, al menos entre el 2005 y el 2009, las organizaciones de la movilización han configurando un entramado que los acerca y que tiende a facilitar su articulación como un movimiento social. 
De acuerdo a su densidad, la red de interacciones supera la red de valoraciones, $11 \%$ y $9 \%$ respectivamente. Esto indica que los vínculos de interacción en comparación a los de valoración tienden a integrar en mayor medida a la movilización. No obstante, el aporte de los vínculos valorativos también es relevante. El efecto conjunto de ambos tipos de vínculos se refleja en la red general de cercanía, la cual alcanza una densidad del $14 \%$.

Lo importante acerca de la densidad de una red va más allá de su valor en sí. Por lo tanto, es fundamental relativizar el valor de la densidad de acuerdo con el tipo de estructura propio de cada red. Así, a pesar de que las anteriores redes posean densidades relativamente bajas, lo importante es que logran integrar a la mayoría de las organizaciones. En efecto, las bajas densidades pueden explicarse por el amplio lapso de tiempo considerado para el estudio: 2005-2009. Este tipo de periodo implica que los vínculos reconocidos por las organizaciones no corresponden de forma exacta, con el recuento exhaustivo de todos sus vínculos de interacción o de valoración; sino que, tienden a resaltar los más relevantes y estables para cada organización. Estas redes representan entonces, una línea de base a partir de la cual su tamaño y su densidad pueden aumentar en los momentos de mayor confrontación social.

En relación a sus componentes, tanto la red de interacciones como la de valoraciones se dividen en un componente principal que agrupa a la mayoría de las organizaciones y un conjunto adicional de organizaciones aisladas. Esto indica que ambas redes son inconexas y no alcanzan a integrar a la totalidad de las organizaciones. De hecho, la red de interacciones se divide en tres componentes: el principal y las dos organizaciones aisladas; y de la misma forma, la red de valoraciones se divide en cinco.

Los anteriores resultados permiten comenzar con el análisis de la posición estructural de las organizaciones con el grupo conformado por los componentes aislados. En este caso, el aislamiento implica que un grupo de organizaciones a pesar de trabajar con una o varias de las problemáticas del agua en la región, tienden a no establecer vínculos directos con las otras organizaciones de la movilización. No obstante, esto no implica que estén totalmente aisladas puesto que pueden estar vinculadas de forma indirecta a través del entorno organizativo. Lo cual se aborda en la siguiente sección. Sin embargo, por ahora, la principal consecuencia de estar aislado es carecer de poder relacional. Es decir, son actores sin capacidad para influenciar los flujos materiales y simbólicos en la red y, por lo tanto, tienden a no poseer impacto alguno sobre los procesos colectivos necesarios para el movimiento social. 
En primer lugar, dos organizaciones están totalmente desconectadas del resto de la movilización, tanto en términos de interacción como de valoración, estas son: el Instituto Internacional de Recursos Renovables A.C. - México, y el Patronato ProMazahua A.C. Estas corresponden a los dos puntos aislados en la margen superior izquierda en la red general de cercanía 2005-2009 (gráfico 3). En segundo lugar, otras dos organizaciones están aisladas parcialmente puesto que, a pesar tener vínculos de interacción con la movilización carecen de vínculos de valoración, estas son: la Red Ecologista Autónoma de la Cuenca de México - Ecocomunidades, y la Fundación Tlaloc A.C. La diferencia entre las primeras y las segundas es que, mientras las primeras tienden a no tener poder relacional alguno, las segundas, aunque están aisladas en la red de valoraciones, poseen un rol periférico y así, algo de poder relacional en la red de interacciones.

De una parte, las organizaciones totalmente aisladas pueden relacionarse con el hecho de que ambas trabajan enfocadas en la asistencia directa a poblaciones específicas: de una parte, el Instituto Internacional de Recursos Renovables A.C. brinda capacitación a poblaciones de bajos y medios recursos económicos sobre el uso de ecotecnologías relacionadas con el agua. De otra parte, el Patronato ProMazahua A.C. apoya el mejoramiento de la calidad de vida de la comunidad Mazahua. En efecto, la participación en acciones colectivas y la configuración de redes sociales con otras organizaciones no hacen parte de sus prioridades básicas. De otra parte, las organizaciones parcialmente aisladas, como la Red Ecologista Autónoma de la Cuenca de México - Ecocomunidades y de la Fundación Tlaloc A.C., serán abordadas más adelante junto al resto de organizaciones que comparten las posiciones más periféricas.

En el componente principal de las redes se observa que algunas organizaciones cuentan con múltiples vínculos y ocupan las posiciones más centrales, mientras que otras se encuentran en las posiciones más periféricas con una menor proporción de conexiones. En primer lugar, la relevancia de los actores centrales consiste en ser la fuente principal de integración y liderazgo al interior de la movilización. En tal medida, poseen los mayores grados de control sobre los flujos materiales y simbólicos, impulsan la formación y el desarrollo de las redes, e impactan con mayor potencia sobre los procesos colectivos de la movilización. En segundo lugar, las organizaciones más periféricas representan a los potenciales actores antagonistas y/o complementarios frente a las creencias y las prácticas dominantes difundidas por lo actores centrales. En este sentido, configuran una fuente potencial de contraste y de debate al interior de la movilización. De manera adicional, regulan el nivel de centralización de las redes. Es decir que, a mayor cantidad de 
vínculos entre las organizaciones periféricas es menor el nivel de centralización en la red $y$, por lo tanto, el poder relacional tiende a distribuirse de forma más homogénea. Dicha homogeneidad es relevante y se relaciona con el desarrollo de procesos colectivos que tienden a ser menos autoritarios y más democráticos.

La estructura del componente principal en las redes observadas es parcialmente segmentada y se caracteriza por múltiples liderazgos o centralidades. Ser parcialmente segmentados significa que la distancia que separa a las organizaciones entre sí es variable. La distancia se corresponde con la cantidad de vínculos que se deben recorrer desde una hasta conectarse con cualquier otra. En este sentido, algunas organizaciones funcionan como puntos de paso obligado, es decir, como intermediarios que vinculan indirectamente a otras. Sin embargo, como se puede apreciar en los gráficos 1,2 y 3, la existencia de múltiples vínculos periféricos evidencia un esfuerzo importante para evitar que la integración de la movilización dependa exclusivamente de unas pocas organizaciones centrales. Dicho esfuerzo se traduce en que las redes adquieren una estructura más homogénea, en la cual, el poder relacional está menos centralizado.

En la tabla a continuación se encuentran las organizaciones clasificadas de acuerdo a sus posiciones estructurales (excluyendo a las dos organizaciones totalmente aisladas). En frente de su nombre se encuentra el valor relativo de su centralidad de grado en cada una de las redes analizadas. Este porcentaje indica la proporción de vínculos de una organización en relación al resto de la movilización. De tal forma, el $0 \%$ es la centralidad de las organizaciones aisladas, mientras que el $100 \%$ correspondería a las organizaciones que se vinculan con todas las otras. Las organizaciones más centrales en cada una de las redes están resaltadas en casillas amarillas. El resto de actores en casillas grises corresponden con las organizaciones más periféricas al interior de la movilización.

\begin{tabular}{|c|c|c|c|c|}
\hline No. & Nombre de la organización & $\begin{array}{l}\text { Red General } \\
\text { de Cercanía }\end{array}$ & $\begin{array}{c}\text { Red de } \\
\text { Interacción }\end{array}$ & $\begin{array}{l}\text { Red de } \\
\text { Valoración }\end{array}$ \\
\hline 8 & $\begin{array}{l}\text { CEMDA A.C. Centro Mexicano de } \\
\text { Derecho Ambiental }\end{array}$ & $53 \%$ & $41 \%$ & $35 \%$ \\
\hline 5 & $\begin{array}{l}\text { COMDA. Coalición de Organizaciones } \\
\text { Mexicanas por el Derecho al Agua }\end{array}$ & $47 \%$ & $41 \%$ & $35 \%$ \\
\hline 10 & Red Mexicana de Acción por el Agua & $41 \%$ & $41 \%$ & $18 \%$ \\
\hline 13 & Guardianes de los Volcanes A.C. & $41 \%$ & $29 \%$ & $35 \%$ \\
\hline 1 & $\begin{array}{l}\text { Fondo para la Comunicación y la } \\
\text { Educación Ambiental A.C. }\end{array}$ & $35 \%$ & $6 \%$ & $35 \%$ \\
\hline 4 & Consejo Consultivo del Agua A.C. & $29 \%$ & $29 \%$ & $18 \%$ \\
\hline 17 & Equipo Pueblo A.C. & $29 \%$ & $29 \%$ & $12 \%$ \\
\hline 11 & $\begin{array}{l}\text { CEJA A.C. Centro de Estudios } \\
\text { Jurídicos Ambientales }\end{array}$ & $24 \%$ & $18 \%$ & $18 \%$ \\
\hline
\end{tabular}




\begin{tabular}{|c|c|c|c|c|}
\hline 14 & $\begin{array}{ll}\text { UPREZ. Unión } & \text { Popular } \\
\text { Revolucionaria Emiliano Zapata }\end{array}$ & $24 \%$ & $24 \%$ & $12 \%$ \\
\hline 16 & Mujer y Medio Ambiente A.C. & $24 \%$ & $12 \%$ & $18 \%$ \\
\hline 18 & $\begin{array}{l}\text { Fondo Pro-Cuenca del Valle de } \\
\text { Bravo A.C. }\end{array}$ & $24 \%$ & $18 \%$ & $18 \%$ \\
\hline 3 & $\begin{array}{llr}\text { Red Ecologista } & \text { Autónoma de la } \\
\text { Cuenca de } & \text { México } & - \\
\text { Ecocomunidades } & & \\
\end{array}$ & $18 \%$ & $18 \%$ & $0 \%$ \\
\hline 6 & PRONATURA A.C. & $18 \%$ & $18 \%$ & $6 \%$ \\
\hline 7 & Presencia Ciudadana Mexicana A.C. & $18 \%$ & $18 \%$ & $12 \%$ \\
\hline 12 & Fundación Tlaloc A.C. & $6 \%$ & $6 \%$ & $0 \%$ \\
\hline 15 & $\begin{array}{l}\text { Asistencia Legal por los Derechos } \\
\text { Humanos - ASILEGAL A.C. }\end{array}$ & $6 \%$ & $6 \%$ & $6 \%$ \\
\hline
\end{tabular}

Tabla 2. Actores centrales y periféricos de acuerdo a sus indicadores de centralidad de grado en las redes informales al interior de la movilización

En primer lugar, con respecto a los actores centrales, se destacan dos organizaciones que lo son tanto en la red de interacciones como en la de valoraciones: el (CEMDA) Centro Mexicano de Derecho Ambiental A.C., y la (COMDA) Coalición de Organizaciones Mexicanas por el Derecho al Agua. Por una parte, el CEMDA, dedicado a la aplicación de la legislación ambiental, es importante para la difusión, defensa y aplicación del derecho humano al agua. De otra parte, la COMDA, enfocada en el desarrollo de la integración y el apoyo entre las organizaciones sociales que defienden el derecho humano al agua, es clave como organización canalizadora de iniciativas colectivas. Juntas organizaciones juegan un rol central en dos sentidos: primero, como líderes prácticos que estimulan la realización de diversas acciones colectivas y de intercambios de información; y segundo, como líderes simbólicos, al plantear el tema de la defensa del derecho humano al agua como un referente para la identificación colectiva de la movilización.

Las siguientes organizaciones en consideración son centrales en solo una de las dos redes y por lo tanto, aunque relevantes, tienen posiciones estructurales de menor impacto que las anteriores. De una parte, en la red de interacciones, se destaca el rol de la Red Mexicana de Acción por el Agua, organización enfocada en brindar asesoría y fortalecimiento a otras para estimular mayores grados de incidencia política, la cual también es facilitadora para la realización de acciones colectivas. No obstante, su posición en la red de valoraciones es periférica, lo cual indica un perfil enfocado más en la cooperación concreta a través de acciones que en el desarrollo de vínculos subjetivos de reconocimiento. 
De otra parte, en la red de valoraciones, se resaltan tanto los Guardianes de los Volcanes A.C., como el Fondo para la Comunicación y la Educación Ambiental A.C., como referentes simbólicos, dado sus altos niveles de reconocimiento como aliados estratégicos y como fuentes de apoyo. En primer lugar, los Guardianes de los Volcanes A.C. están orientados al desarrollo de procesos de gestión comunitaria de los recursos naturales, además de la generación y la difusión de ecotecnologías aplicadas al agua y a otros recursos naturales. Esta organización aporta algunos referentes adicionales para la identificación colectiva como son el trabajo comunitario y la difusión de ecotecnologías. Por lo tanto, posee un rol clave al ampliar el conjunto de referentes para la identificación colectiva y así, robustecer la integración subjetiva de la movilización.

En segundo lugar, el Fondo para la Comunicación y la Educación Ambiental A.C. cobija un portal electrónico para el encuentro y el intercambio de información entre diversos actores sociales vinculados con las problemáticas del agua a escala local, nacional e internacional (http://agua.org.mx/). Esta organización es central en la red valoraciones al configurar un apoyo general para el flujo de información y la generación de dialogo y debate. En esta medida, representa e impulsa los valores asociados a la democratización de la información sobre el agua y a la necesidad constante de diálogos abiertos e inclusivos entre los actores sociales interesados.

Las cinco organizaciones abordadas anteriormente configuran el grupo de los actores más centrales para la integración de la red general de cercanía 2005-2009. Al liderar el proceso de articulación de la movilización son los más influyentes sobre sus procesos colectivos. Así, a manera de recuento, su influencia se caracteriza por proponer 'el derecho humano al agua' como marco para la interpretación de las diversas problemáticas, favorecer el intercambio de la información y el apoyo a modelos de manejo comunitario del agua y de los recursos naturales. De acuerdo a lo anterior, las acciones colectivas tienden a enfocarse, en primer lugar, en la defensa de dicho derecho y en la búsqueda de incidencia sobre las políticas públicas; y en segundo lugar, en promover procesos locales de organización y capacitación.

En relación con las organizaciones más periféricas, su rol principal es el de desconcentrar el poder relacional en la red. En este sentido, representan las alternativas potenciales de integración que contrastan y matizan la influencia de los actores centrales. A pesar de su menor poder relacional, las organizaciones periféricas aportan un carácter pluralista e incluyente a la movilización. 
Al interior de este grupo se encuentran organizaciones como: el Equipo Pueblo A.C., el Consejo Consultivo del agua A.C., el (CEJA) Centro de Estudios Jurídicos y Ambientales A.C, y Presencia Ciudadana Mexicana A.C., las cuales se enfocan en el fortalecimiento de procesos asociados con la participación social. Otro subgrupo lo configuran Pronatura México A.C. y la Fundación Tlaloc A.C., que realizan proyectos de conservación de los recursos naturales en la región. También se incluyen otras organizaciones como (ASILEGAL) Asistencia Legal por los Derechos Humanos A.C., dedicada a la defensa de los derechos humanos en general; la (UPREZ) Unión Popular Revolucionaria Emiliano Zapata, que aborda los problemas del agua vinculados con el desarrollo urbano sostenible y la adecuada prestación de los servicios públicos; Mujer y Medio Ambiente A.C., que combina el apoyo al desarrollo sustentable con diversas demandas por la equidad de género; y la Red Ecologista Autónoma de la Cuenca de México, que difunde una perspectiva alternativa para la interpretación de las problemáticas del agua en la región basada en el concepto del ecocentrismo.

\section{Las redes informales entre la movilización y su contexto organizativo}

Para el periodo 2005-2009 se identifica un contexto organizativo compuesto por 143 organizaciones clasificadas en seis grupos: 46 organizaciones sociales nacionales, 29 instituciones públicas nacionales, 28 organizaciones sociales internacionales, 20 organizaciones privadas, 13 instituciones educativas nacionales, y 7 instituciones públicas internacionales. Todas estas organizaciones se relacionan con la movilización por el agua en la Cuenca de México por medio de los vínculos de interacción o de valoración planteados anteriormente. Su diversidad indica que el contexto organizativo es multifacético. Es decir que, a pesar de una mayoría de organizaciones sociales nacionales e internacionales que tienden a coincidir con la movilización en su apoyo al derecho humano al agua; también se encuentran diversas instituciones gubernamentales y organizaciones privadas las cuales, por el contrario, tienden a favorecer una visión mercantil del agua. En efecto, todos estos grupos tienen influencia potencial, en mayor o menor medida, sobre el desarrollo de los procesos colectivos de la movilización. 


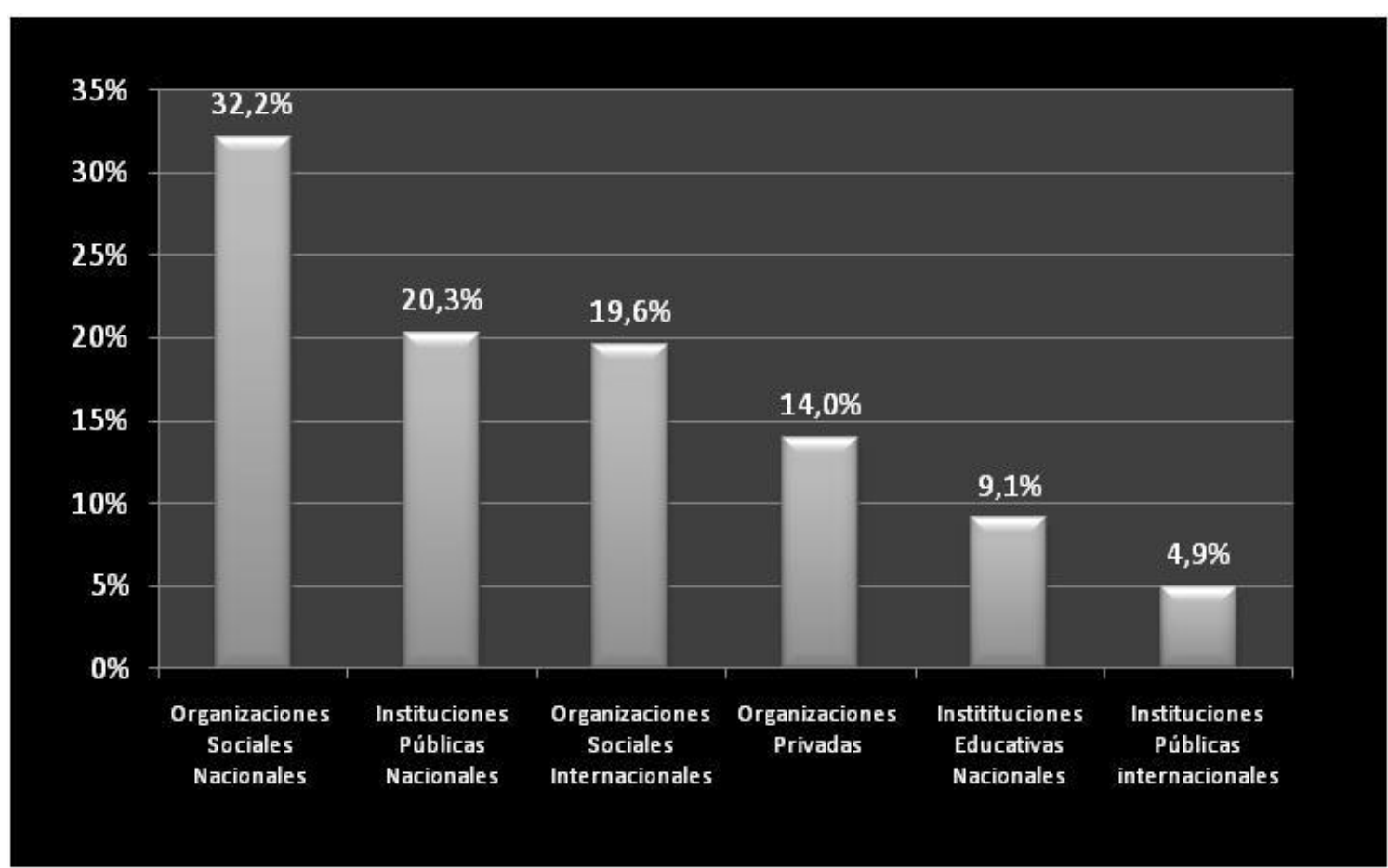

Gráfico 4. Composición del contexto organizativo de la movilización 2005-2009.

El anterior grafico, resume la composición del contexto organizativo de la movilización. Sus resultados evidencian que, en primer lugar, la mayoría del contexto organizativo de la movilización está compuesto por organizaciones sociales nacionales $(32 \%)$. Las cuales, sumadas a las organizaciones sociales internacionales (20\%) corresponden a más del $50 \%$ del total. Las organizaciones nacionales incluyen tanto asociaciones civiles formalmente constituidas, como alianzas, asambleas, asociaciones, coaliciones, movimientos y uniones, las cuales tienden a permanecer informales. De otra parte, las organizaciones internacionales incluyen redes y fundaciones que en su mayoría apoyan, estimulan y coordinan el trabajo de diversas organizaciones sociales locales y nacionales. Estos dos grupos, en conjunto, indican que la movilización se acerca de manera principal a otras organizaciones relacionadas directa e indirectamente con el agua en todos los ámbitos geográficos. Así mismo, confirma que los problemas relacionados con el agua configuran temas de relevancia social creciente que tienden a movilizar y a estimular el acercamiento entre organizaciones sociales desde lo local hasta lo global. 
En cuanto a su impacto sobre la movilización, las organizaciones sociales nacionales e internacionales tienden a promover la defensa del agua interpretada como un bien común y como derecho humano, a denunciar diversas inequidades asociadas a su acceso y uso, y a apoyar la generación de conciencia ambiental y de capacidades entre diversas poblaciones locales para fomentar su sostenibilidad, entre otras causas. Esta influencia reafirma las interpretaciones dominantes dentro de la movilización y sirven para enmarcar las problemáticas locales del agua en la Cuenca de México en una problemática mayor de alcance nacional y global.

Las instituciones públicas nacionales: locales, estatales y federales representan un $20 \%$ del contexto organizativo. Esto revela una cercanía relevante entre la movilización y el sector gubernamental. En efecto, el sector gubernamental es una de las principales fuentes de financiación para la ejecución de proyectos tanto sociales como ambientales por parte de la movilización. Las principales influencias desplegadas desde el sector gubernamental sobre la movilización incluyen tanto su apoyo al discurso del desarrollo sustentable como sus prácticas que tienden a favorecer la visión mercantilista del agua, entendida como un recurso natural. Según lo anterior, la movilización despliega una estrategia que integra un balance complejo entre la cooperación y la oposición frente a las autoridades gubernamentales.

Con una influencia similar a la gubernamental, basada en una visión mercantil del agua y matizada por el concepto de desarrollo sostenible, las organizaciones privadas corresponden al $14 \%$ del contexto organizativo. Este grupo incluye principalmente fundaciones empresariales que a través de sus programas de responsabilidad social y ambiental ofrecen recursos financieros para apoyar el diseño y la ejecución de proyectos relacionados con el agua en la región.

Por último, se encuentran los grupos conformados por las instituciones educativas y las instituciones públicas internacionales, las cuales representan el $9 \%$ y el $5 \%$ del contexto organizativo. Estos son los grupos con menor participación y, por lo tanto, con menor influencia sobre los procesos colectivos de la movilización. De una parte, las instituciones educativas tienen a vincularse con la movilización a través de investigadores o centros de investigación específicos con intereses puntuales en las problemáticas del agua a escala local y nacional. El principal nexo se basa tanto en el intercambio de información para los estudios realizados por los investigadores y la retroalimentación que sus resultados brindan a la movilización, como en la realización de eventos académicos que estimulan el encuentro y el debate entre diversos actores sociales interesados en la materia. De otra parte, las instituciones públicas internacionales son principalmente fuentes de recursos financieros, no 
obstante, su relevancia es mínima en comparación de otras fuentes como el sector social internacional o el sector público y privado nacional.

Para pasar de la influencia general por subgrupos hacia la identificación de las organizaciones específicas con mayor impacto sobre los procesos colectivos de la movilización, a continuación se ofrecen una serie de tablas que listan a los actores según los subgrupos que conforman el contexto organizativo de la movilización.

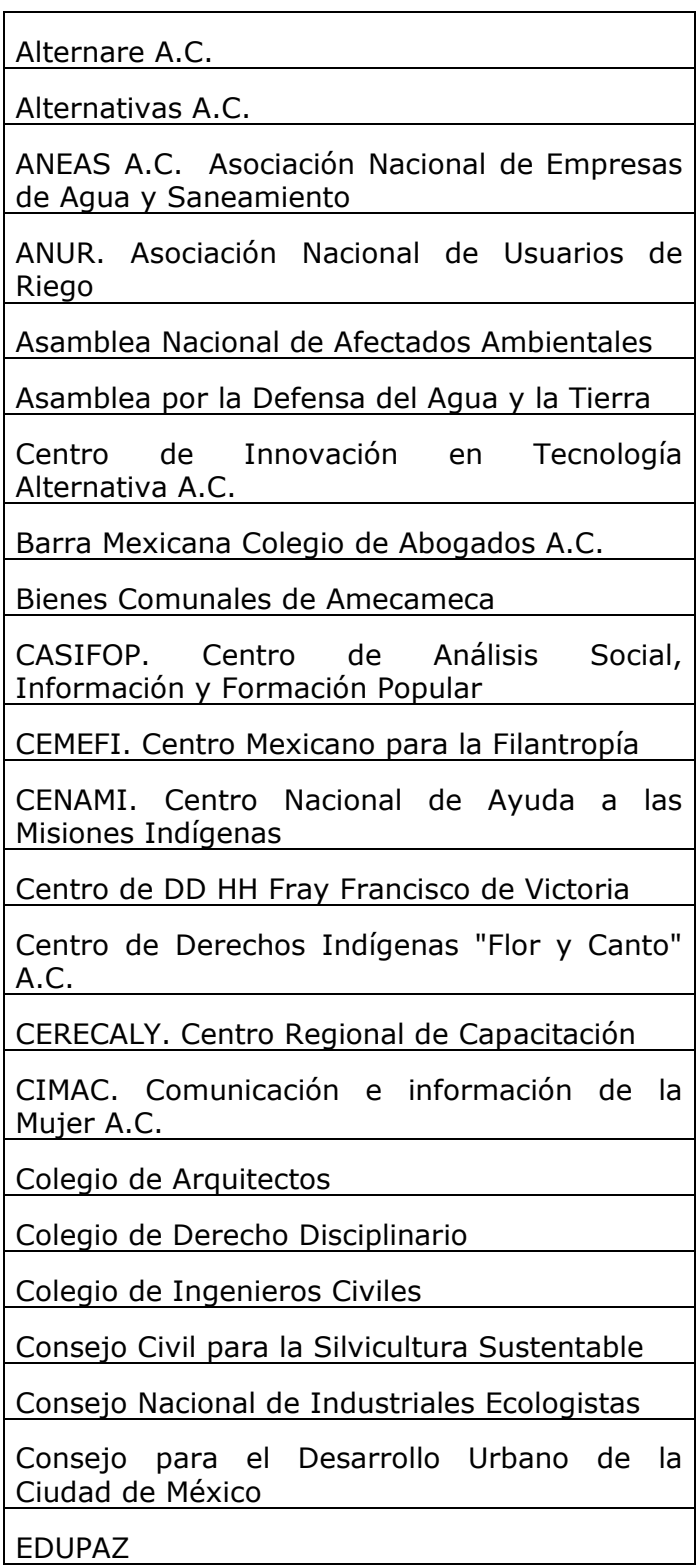

\begin{tabular}{l} 
Fondo Mexicano para la Conservación de la \\
Naturaleza \\
\hline GEA. Grupo de Estudios ambientales \\
\hline Grupedsac A.C. \\
\hline IDEA. Instituto de Derecho Ambiental \\
\hline $\begin{array}{l}\text { Ilustre y Nacional Colegio de Abogados de } \\
\text { México }\end{array}$ \\
\hline Iniciativa Mexicana para la Conservación \\
\hline $\begin{array}{l}\text { Jóvenes Independientes Otomíes de San Mateo } \\
\text { de Capulhuac }\end{array}$ \\
\hline Manos a la tierra \\
\hline $\begin{array}{l}\text { MAPDER. Movimiento Mexicano de Afectados } \\
\text { por las Presas y en Defensa de los Ríos }\end{array}$ \\
\hline Movimiento de los 13 pueblos de Morelos \\
\hline Movimiento Mazahua \\
\hline Movimiento Urbano Popular \\
\hline Mundo Sustentable A.C. \\
\hline Pacto de Grupos Ecologistas \\
\hline Patronato Pro-Valle \\
\hline Procoyoacán \\
\hline Pro-Fauna \\
\hline $\begin{array}{l}\text { RADAR. Colectivo de Estudios Críticos en } \\
\text { Derecho }\end{array}$ \\
\hline Red por una Nueva Cultura del Agua \\
\hline REGEMA. Red Genero y Medio Ambiente \\
\hline $\begin{array}{l}\text { RMALC. Red Mexicana de Acción contra el Libre } \\
\text { Comercio }\end{array}$ \\
\hline SENDAS A.C. \\
Somos Mundo \\
\hline
\end{tabular}

Tabla 3. Organizaciones sociales nacionales. 


\begin{tabular}{|l|}
\hline $\begin{array}{l}\text { AIDA. Asociación Interamericana de de } \\
\text { Defensa Ambiental }\end{array}$ \\
\hline BirdLife International \\
\hline Both Ends \\
\hline Climate Action Network \\
\hline Council of Canidians \\
\hline Desarrollo y Paz \\
\hline FIAN. Foodfirst Information \& Action Network \\
\hline $\begin{array}{l}\text { FLACAM. Foro Latinoamericano de Ciencias } \\
\text { Ambientales }\end{array}$ \\
\hline Foro Social Mundial \\
\hline Green Peace \\
\hline GYAN. Global Youth Action Network \\
\hline $\begin{array}{l}\text { HIC. Coalición Internacional por el Hábitat } \\
\text { México }\end{array}$ \\
\hline
\end{tabular}

IDEX - International Development Exchange

OXFAM. Oxford Commitee for Famine Relief

Pan para el Mundo

Red Centroamericana de Acción por el Agua

Red Social Watch

Red VIDA

The Climate Proyect

The Hewlett Foundation

The Resource Foundation

The World Resources Institute

Tribunal Latinoamericano del Agua - TLA

UUSC. Unitarian Universalist Service Committee

Water Aid

Waterkeeper Alliance

WWF. World Wildlife Fund

Tabla 4. Organizaciones sociales internacionales.

\begin{tabular}{l} 
Cámara Mexicana de Industria de la \\
Construcción \\
\hline $\begin{array}{l}\text { CEPANAF. Comisión Estatal de Parques } \\
\text { Naturales y de la Fauna }\end{array}$ \\
\hline Colegio de Posgraduados \\
\hline $\begin{array}{l}\text { Comisión de Derechos Humanos del Distrito } \\
\text { Federal }\end{array}$ \\
\hline $\begin{array}{l}\text { Comisión de Gestión Integral del Agua de la } \\
\text { Asamblea Legislativa }\end{array}$ \\
\hline CNA. Comisión Nacional del Agua \\
\hline CONAFOR. Comisión Nacional Forestal \\
\hline Gobierno del Distrito Federal \\
\hline Gobierno del Estado de México \\
\hline Gobierno Federal \\
\hline $\begin{array}{l}\text { IMTA. Instituto Mexicano de Tecnologías del } \\
\text { Agua }\end{array}$ \\
\hline $\begin{array}{l}\text { INDESOL. Instituto Nacional de Desarrollo } \\
\text { Social }\end{array}$ \\
\hline Instituto de Agua de Nuevo León \\
\hline Instituto de las Mujeres del DF \\
\hline INE. Instituto Nacional de Ecología \\
\hline Instituto Nacional de las Mujeres \\
\hline
\end{tabular}

Institutos Delegaciones de las Mujeres

INVI. Instituto de Vivienda del Distrito Federal

JAPEM, Junta de Asistencia Privada del Estado de México

PROBOSQUE Estado de México

PAOT. Procuraduría Ambiental y de Ordenamiento Territorial del D.F.

PROFEPA. Procuraduría Federal de Protección al Ambiente

SAGARPA. Secretaria de Agricultura, Ganadería, Desarrollo Rural, Pesca y Alimentación

Secretaría de Desarrollo Social de Distrito Federal

Secretaría de Gobierno del Distrito Federal

Secretaría de Medio Ambiente del Estado de México

SEDEREC. Secretaría de Desarrollo Rural y Equidad para las Comunidades

SEMARNAT. Secretaría del Medio Ambiente y Recursos Naturales.

SACM. Sistema de Aguas de la Ciudad de México

Tabla 5. Instituciones públicas nacionales. 


\begin{tabular}{|l|}
\hline ALSEA \\
\hline CORE Water Managment Solutions \\
\hline Fomento Ecológico BANAMEX \\
\hline Fundación Bancomer \\
\hline Fundación Coca-Cola \\
\hline Fundación FEMSA \\
\hline Fundación Gonzalo Río Arronte \\
\hline Fundación Grupo Modelo \\
\hline Fundación Heinrich Boll \\
\hline Fundación Helvex \\
\hline
\end{tabular}

Fundación Pedro y Helena Hernández

Fundación Rockefeller

Fundación Televisa

Fundación Wall Mart

Genoma Lab

Grupo Bimbo

Procter and Gamble

Revista Teorema del Agua

SARAR Transformación

Supermercado Supercompras

Unión de Grupos Ambientalistas

Tabla 6. Organizaciones privadas.

\begin{tabular}{|l|}
\hline $\begin{array}{l}\text { Centro de Capacitación para el Desarrollo } \\
\text { Empresarial }\end{array}$ \\
\hline $\begin{array}{l}\text { CLARES, Centro Latinoamericano de } \\
\text { Responsabilidad Social, Universidad Anáhuac }\end{array}$ \\
\hline Colegio Mexiquense \\
\hline El Colegio de México A.C. \\
\hline $\begin{array}{l}\text { FLACSO. Facultad Latinoamericana de Ciencias } \\
\text { Sociales }\end{array}$ \\
\hline Instituto Politécnico Nacional \\
\hline Instituto Tecnológico de Toluca \\
\hline
\end{tabular}

Instituto Tecnológico de Monterrey

UAEM. Universidad Autónoma del Estado de México

UAM. Universidad Autónoma Metropolitana

UNAM. Universidad Nacional Autónoma de México

Universidad Autónoma Chapingo

Universidad Iberoamericana

Tabla 7. Instituciones educativas.

\begin{tabular}{|l|}
\hline Banco Mundial \\
\hline Centro de información de la ONU \\
\hline Embajada Británica \\
\hline Embajada Suiza \\
\hline
\end{tabular}

EPA. Environmental Protection Agency

OEA. Organización de Estados Américanos

Unesco

Tabla 8. Instituciones públicas internacionales.

Al comparar los grafos 5 y 6 a continuación, se pueden identificar a los actores más centrales del contexto organizativo de la movilización. Los actores centrales del contexto poseen el rol de intermediarios y de generadores de vínculos indirectos entre las organizaciones de la movilización. Así, mientras el grafo 5 corresponde con la red general de cercanía 2005-2009 que incluye a los 143 acores del contexto representados con puntos negros y a las 18 organizaciones de la movilización con puntos rojos. En el grafo 6 se excluyen los actores del contexto vinculados con solo una organización de la movilización y permanecen aquellos conectados con dos o más. De tal forma, el grafo 6 simboliza a la red de vínculos indirectos de la movilización 2005-2009, en función de su contexto organizativo. Es decir que, representa la capacidad del contexto organizativo para integrar de forma indirecta a las organizaciones al interior de la movilización. En ambas redes, 5 y 6, las líneas 
representan el reconocimiento de al menos uno de los vínculos de cercanía planteados anteriormente.

De acuerdo al grafo 6, este subgrupo de actores del contexto integra de forma indirecta a todas las organizaciones de la movilización en un solo componente. Esto indica que las organizaciones de la movilización poseen un contexto organizativo compartido. En el cual, al menos 34 de los 143 actores del contexto, es decir el $24 \%$ del total, se conectan con dos o más organizaciones de la movilización. Esta red también muestra que los actores del contexto ocupan posiciones estructurales diversas, más centrales o periféricas. La diferencia consiste en que las más centrales poseen los mayores grados de poder relacional y, por lo tanto, tienen el mayor control para la intermediación y para influenciar sobre los procesos colectivos de la movilización. Mientras que las más periféricas desconcentran la red y por lo tanto representan a las influencias alternativas y potenciales del contexto.

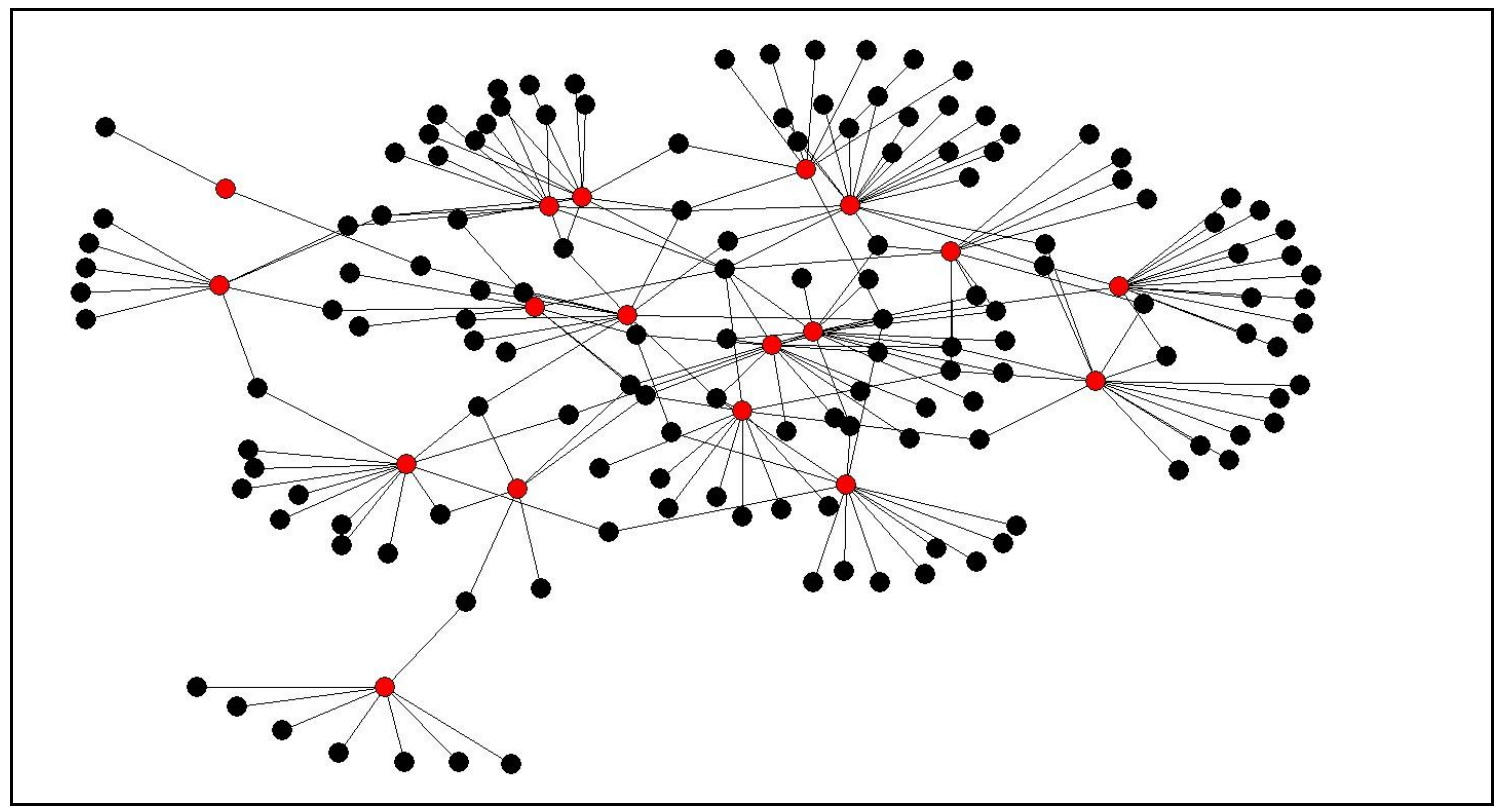

Gráfico 5. Red general de cercanía 2005-2009, entre la movilización y su contexto organizativo. 


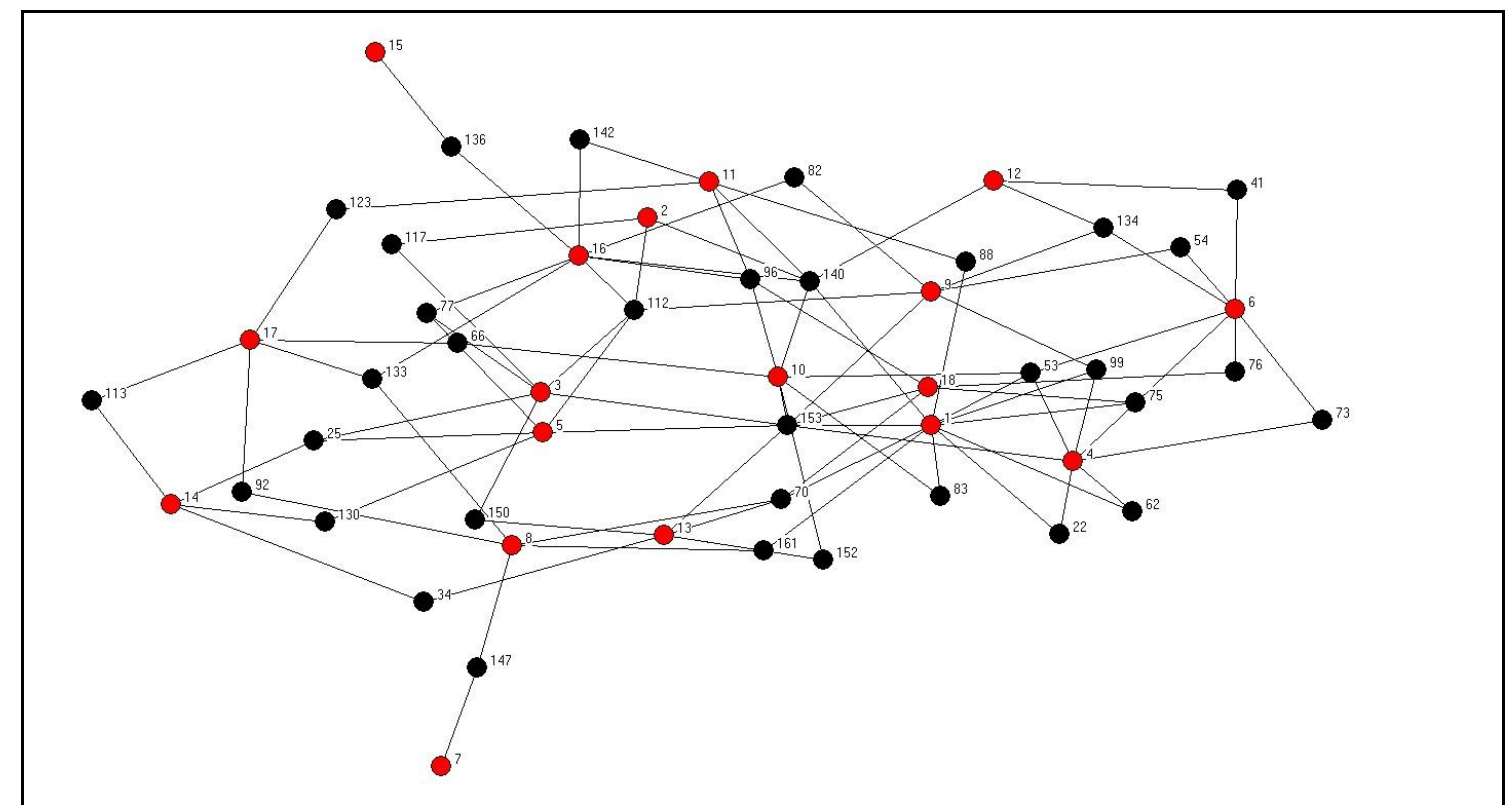

Gráfico 6. Red general de cercanía 2005-2009, vínculos indirectos de la movilización por medio del contexto organizativo.

En este sentido, comenzando por los actores más centrales del contexto, la siguiente tabla los enuncia junto con su grado de centralidad. En la columna anterior al nombre se encuentra el número que sirve para identificar a cada actor en el grafo 6.

\begin{tabular}{|c|l|l|c|}
\hline No. & \multicolumn{1}{|c|}{ Nombre } & \multicolumn{1}{c|}{ Grupo } & Centralidad \\
\hline $\begin{array}{c}15 \\
3\end{array}$ & UNAM. Universidad Nacional Autónoma de México & $\begin{array}{l}\text { Instituciones } \\
\text { Educativas }\end{array}$ & $44 \%$ \\
\hline $\begin{array}{c}14 \\
0\end{array}$ & $\begin{array}{l}\text { SEMARNAT. Secretaria del Medio Ambiente y Recursos } \\
\text { Instituciones Públicas }\end{array}$ & $33 \%$ \\
\hline $\begin{array}{c}11 \\
2\end{array}$ & Movimiento Mazahua & $\begin{array}{l}\text { Organizaciones } \\
\text { Sociales Nacionales }\end{array}$ & $28 \%$ \\
\hline 70 & Fondo Mexicano para la Conservación de la Naturaleza & $\begin{array}{l}\text { Organizaciones } \\
\text { Sociales Nacionales }\end{array}$ & $22 \%$ \\
\hline 75 & Fundación Gonzalo Río Arronte & $\begin{array}{l}\text { Organizaciones } \\
\text { Privadas }\end{array}$ & $22 \%$ \\
\hline 53 & CNA. Comisión Nacional del Agua & Instituciones Públicas & $22 \%$ \\
\hline 96 & IMTA. Instituto Mexicano de Tecnologías del Agua & Instituciones Públicas & $22 \%$ \\
\hline
\end{tabular}

Tabla 9. Organizaciones del contexto con mayores grados de centralidad.

Según la anterior tabla, el grupo de los actores más centrales incluye a tres instituciones públicas, dos organizaciones sociales nacionales, una organización privada y una institución educativa. Esto revela, en primer lugar, que la movilización tiende a acercarse principalmente hacia el sector público, representado por algunas de las principales autoridades en relación al manejo del agua en la región, como son: la Comisión Nacional del Agua, la Secretaria del Medio Ambiente y Recursos Naturales, y el Instituto Mexicano de Tecnologías del Agua. Por lo tanto, 
el sector público representa no solo un oponente para la movilización como receptor de sus acciones conflictivas; sino que también configura una fuente de apoyo con influencia sobre el desarrollo de sus procesos colectivos. Según Blühdorn (2006) y Pellow (1999) desde la década de 1980 el desarrollo de diversos movimientos sociales y ambientales a escala global, se ha caracterizado por la realización de múltiples acciones de colaboración especialmente en momentos de crisis sociales, económicas y políticas. Éste tipo de actividad colectiva ha sido importante para trasmitir a la sociedad mensajes y símbolos sobre la confiabilidad, la sostenibilidad y el apoyo a la democracia participativa. Esta observación adquiere relevancia al recordar que, hace al menos una década que en la $\mathrm{CM}$ se ha fortalecido una percepción generalizada de estar enfrentando un momento de crisis e incertidumbre creciente en la situación del agua en la región.

Otra influencia principal para la movilización corresponde a las organizaciones sociales nacionales como son: el Movimiento Mazahua y el Fondo Mexicano para la Conservación de la Naturaleza. Las anteriores, en conjunto con la organización privada la Fundación Gonzalo Rio Arronte representan los esfuerzos de la movilización para mantenerse conectada con el sector social. Así, revela un interés colectivo por contar con los recursos relacionales, materiales y simbólicos, capaces de sustentar el desarrollo de la movilización y de brindarle una cierta autonomía frente al sector público gubernamental.

Las anteriores organizaciones permiten ajustar los límites de la movilización. En efecto, algunos de los actores del contexto comparten las mismas características básicas del conjunto de las 18 organizaciones de la movilización. Es decir, son organizaciones sociales independientes que coinciden en su interés por el agua en la región. Entre estas se encuentra el Movimiento Mazahua que representa la lucha de la comunidad Mazahua frente a los efectos negativos derivados de la gestión del agua en sus territorios y reclamando el respeto a sus derechos humanos. Así mismo, el Fondo Mexicano para la Conservación de la Naturaleza y la Fundación Gonzalo Rio Arronte, son organizaciones que patrocinan a escala nacional la ejecución de proyectos vinculados con el agua. Por lo tanto, en estos casos, más que actores del contexto pueden considerarse como organizaciones que hacen parte integral de la movilización.

Por último, pero no menos importante, esta la Universidad Nacional Autónoma de México. Esta representa la cercanía de la movilización con sector académico, lo cual revela que la movilización tiende a apoyarse también en la información y el conocimiento generado por éste sector. Estos recursos son importantes en tanto 
brindan legitimidad a gran parte de la información que sustenta el desarrollo de procesos colectivos como la generación de significados compartidos.

De forma complementaria, la tabla a continuación presenta a las organizaciones del contexto menos centrales pero con potencialidad de generar vínculos indirectos al interior de la movilización. Como se planteo anteriormente, estas tienden a desconcentrar la red y por lo tanto representan a las influencias alternativas y potenciales del contexto.

\begin{tabular}{|c|c|c|c|}
\hline No. & Nombre & Grupo & Centralidad \\
\hline 25 & Asamblea por la Defensa del Agua y la Tierra & $\begin{array}{l}\text { Organizaciones } \\
\text { Sociales Nacionales }\end{array}$ & $17 \%$ \\
\hline 133 & $\begin{array}{l}\text { RMALC. Red Mexicana de Acción contra el Libre } \\
\text { Comercio }\end{array}$ & $\begin{array}{l}\text { Organizaciones } \\
\text { Sociales Nacionales }\end{array}$ & $17 \%$ \\
\hline 161 & WWF. World Wildlife Fund & $\begin{array}{l}\text { Organizaciones } \\
\text { Sociales } \\
\text { Internacionales }\end{array}$ & $17 \%$ \\
\hline 77 & Fundación Heinrich Boll & $\begin{array}{l}\text { Organizaciones } \\
\text { Privadas }\end{array}$ & $17 \%$ \\
\hline 134 & $\begin{array}{l}\text { SAGARPA. Secretaria de Agricultura, Ganadería, } \\
\text { Desarrollo Rural, Pesca y Alimentación }\end{array}$ & Instituciones Públicas & $17 \%$ \\
\hline 99 & Instituto Politécnico Nacional & $\begin{array}{l}\text { Instituciones } \\
\text { Educativas }\end{array}$ & $17 \%$ \\
\hline 22 & $\begin{array}{l}\text { ANEAS A.C. Asociación Nacional de Empresas de Agua } \\
\text { y Saneamiento }\end{array}$ & $\begin{array}{l}\text { Organizaciones } \\
\text { Sociales Nacionales }\end{array}$ & $11 \%$ \\
\hline 34 & $\begin{array}{l}\text { CASIFOP. Centro de Análisis Social, Información y } \\
\text { Formación Popular }\end{array}$ & $\begin{array}{l}\text { Organizaciones } \\
\text { Sociales Nacionales }\end{array}$ & $11 \%$ \\
\hline 83 & GEA. Grupo de Estudios ambientales & $\begin{array}{l}\text { Organizaciones } \\
\text { Sociales Nacionales }\end{array}$ & $11 \%$ \\
\hline 113 & Movimiento Urbano Popular & $\begin{array}{l}\text { Organizaciones } \\
\text { Sociales Nacionales }\end{array}$ & $11 \%$ \\
\hline 117 & Pacto de Grupos Ecologistas & $\begin{array}{l}\text { Organizaciones } \\
\text { Sociales Nacionales }\end{array}$ & $11 \%$ \\
\hline 66 & FIAN. Foodfirst Information \& Action Network & $\begin{array}{l}\text { Organizaciones } \\
\text { Sociales } \\
\text { Internacionales }\end{array}$ & $11 \%$ \\
\hline 88 & Green Peace & $\begin{array}{l}\text { Organizaciones } \\
\text { Sociales } \\
\text { Internacionales }\end{array}$ & $11 \%$ \\
\hline 92 & HIC. Coalición Internacional por el Hábitat México & $\begin{array}{l}\text { Organizaciones } \\
\text { Sociales } \\
\text { Internacionales }\end{array}$ & $11 \%$ \\
\hline 130 & $\begin{array}{l}\text { Red VIDA. Vigilancia Interamericana para la Defensa y } \\
\text { el Derecho al Agua. }\end{array}$ & $\begin{array}{l}\text { Organizaciones } \\
\text { Sociales } \\
\text { Internacionales }\end{array}$ & $11 \%$ \\
\hline 147 & The Hewlett Foundation & $\begin{array}{l}\text { Organizaciones } \\
\text { Sociales } \\
\text { Internacionales }\end{array}$ & $11 \%$ \\
\hline 150 & Tribunal Latinoamericano del Agua - TLA & $\begin{array}{l}\text { Organizaciones } \\
\text { Sociales } \\
\text { Internacionales }\end{array}$ & $11 \%$ \\
\hline 73 & Fundación Coca-Cola & Organizaciones & $11 \%$ \\
\hline
\end{tabular}




\begin{tabular}{|c|l|l|c|}
\hline 76 & Fundación Grupo Modelo & Privadas & $11 \%$ \\
\hline 82 & Fundación Wall Mart & $\begin{array}{l}\text { Organizaciones } \\
\text { Privadas }\end{array}$ & $11 \%$ \\
\hline 41 & $\begin{array}{l}\text { CEPANAF. Comisión Estatal de Parques Naturales y de } \\
\text { la Fauna }\end{array}$ & Instituciones Públicas & $11 \%$ \\
\hline 54 & CONAFOR. Comisión Nacional Forestal & Instituciones Públicas & $11 \%$ \\
\hline 123 & $\begin{array}{l}\text { PAOT. Procuraduría Ambiental y de Ordenamiento } \\
\text { Territorial del D.F. }\end{array}$ & Instituciones Públicas & $11 \%$ \\
\hline 136 & Secretaría de Desarrollo Social DF & Instituciones Públicas & $11 \%$ \\
\hline 142 & Sistema de Aguas de la Ciudad de México SACM & Instituciones Públicas & $11 \%$ \\
\hline 62 & El Colegio de México A.C. & $\begin{array}{l}\text { Instituciones } \\
\text { Educativas }\end{array}$ & $11 \%$ \\
\hline 152 & UAM. Universidad Autónoma Metropolitana & $\begin{array}{l}\text { Instituciones } \\
\text { Educativas }\end{array}$ & $11 \%$ \\
\hline
\end{tabular}

Tabla 10. Organizaciones del contexto con menores grados de centralidad, que generan vínculos indirectos al interior de la movilización.

Este grupo se compone de 27 actores, entre los cuales siete son organizaciones sociales nacionales, siete son organizaciones sociales internacionales, seis son instituciones públicas, cuatro son organizaciones privadas y tres son instituciones educativas.

Estos resultados señalan que, primero, se confirma el esfuerzo por parte de la movilización para relacionarse con otras organizaciones sociales nacionales y, así, desarrollar sus procesos colectivos con cierta autonomía frente a la amplia influencia del sistema político. Algunas organizaciones cercanas a la movilización en este sentido son: la Red Mexicana de Acción frente al Libre Comercio, el Centro de Análisis Social, Información y Formación Popular, el Movimiento Urbano Popular, y el Grupo de Estudios Ambientales.

También se encuentran otras organizaciones sociales que representan el nexo entre la movilización y el movimiento global por el agua. La presencia de éstas es relevante puesto que ofrecen un apoyo simbólico, estimulando la percepción de hacer parte de un proceso colectivo de alcance y relevancia global. Entre estas se encuentran: la Coalición Internacional por el Hábitat, la Red Vida. Vigilancia Interamericana para la Defensa y el Derecho al Agua, y el Tribunal Latinoamericano del Agua.

Se reafirma la cercanía entre la movilización y el sector público representado por seis instituciones públicas nacionales: el Sistema de Aguas de la Ciudad de México, la Comisión Nacional Forestal, la Comisión Estatal de Parques Naturales y de la Fauna, la Procuraduría Ambiental y de Ordenamiento Territorial del Distrito Federal, 
la Secretaría del Desarrollo Social del Distrito Federal, y la Secretaria de Agricultura, Ganadería, Desarrollo Rural, Pesca y Alimentación.

Se identifica una tendencia alternativa basada en la conexión entre una parte de la movilización y algunas organizaciones privadas como la Fundación Wall Mart, la Fundación Grupo Modelo, y la Fundación Coca Cola. Estos actores configuran una influencia controversial al interior de la movilización en tanto que, de una parte, representan a los grupos comerciales que apoyan el desarrollo de una visión mercantil del agua. Mientras que de otra parte, ofrecen recursos para la ejecución de proyectos sociales y ambientales a través de sus programas y obligaciones de responsabilidad social y ambiental. Así, se observa que la movilización tiende a apoyarse en dichos recursos, a pesar de oponerse en general a una visión mercantil del agua. En el caso de la Fundación Heinrich Boll, su enfoque social la diferencia de las anteriores y la acerca al caso de las organizaciones sociales internacionales que apoyan el desarrollo de la movilización nacional y local por el agua.

Por último, aparecen otras tres instituciones educativas: el Instituto Politécnico Nacional, la Universidad Autónoma Metropolitana, y El Colegio de México. Su presencia confirma la tendencia a mantener vínculos con el sector académico. En efecto, este sector ofrece un apoyo para el desarrollo de sus procesos colectivos, en especial, aquellos relacionados con la construcción de significados compartidos en relación al diagnóstico de la problemática local, nacional e internacional; y con la realización de eventos que reúnen a diversos actores sociales interesados en el tema. En suma, la influencia de los actores menos centrales del contexto tiende a reafirmar y a ampliar las influencias de los actores más centrales presentados anteriormente.

A manera de conclusión general, los resultados del análisis aplicado a las redes de la movilización por el agua en la CM entre 2005 y 2009, brindaron la evidencia suficiente para reconocer un proceso de configuración efectiva de una red social, que ha integrado a las 18 organizaciones sociales alrededor de un proyecto colectivo y de un contexto organizativo común. Las estructuras relacionales subyacentes a la acción social de estas organizaciones permitieron en efecto, caracterizarlas no solo como un actor social colectivo sino también como una movilización social en desarrollo, desde una perspectiva teórica basada en su análisis estructural. 
Las redes observadas demostraron que el grupo de organizaciones entrevistadas posee una estructura relacional colectiva capaz de sustentar la realización de diversos tipos de acciones colectivas y que además, es suficiente para relacionarse con y sobrevivir a sus principales contradictores: los actores gubernamentales y empresariales. De manera adicional, estas redes señalan que el grupo posee la potencialidad para sostener y estimular los procesos colectivos fundamentales para el desarrollo de los movimientos sociales.

\section{Bibliografía}

Blühdorn, Ingolfur (2006). "Self-Experience in the theme park of radical action? Social movements and political articulation in the late-modern condition". en European Journal of Social Theory, 9 (1):23-42.

Borgatti, Stephen et al. (2002). Ucinet for Windows: Software for Social Network Analysis. Harvard: Analytic Technologies.

Diani, Mario (2004). "Organizations, coalitions, and movements" en Theory and Society. Vol. 33. No. 3/4, Jun.-Ago. pp. 281-309.

Diani, Mario (2003). "Social movements, contentious actions, and social networks: from metaphor to substance?" en Social movements and networks, relational approaches to collective action. Oxford: Oxford University Press.

Melucci, Alberto (1999). Acción colectiva, vida cotidiana y democracia. México: El Colegio de México.

Pellow, David (1999). "Framing emerging environmental movement tactics: mobilizing consensus, demobilizing conflict", en Sociological Forum, vol. 14, núm. 4, pp. 659-683.

Tarrow, Sidney (2004). El poder en movimiento: los movimientos sociales, la acción colectiva y la política. Madrid: Alianza Editorial.

Tilly, Charles (1978). From Mobilization to Revolution. McGraw-Hill Companies.

Wasserman, Stanley y Katherine Faust (1994). Social network analysis. Methods and applications. Cambridge: Cambridge University press. 